\title{
Application of Expert System in the Analysis of Aluminum Reduction Cell
}

\author{
Miao $\mathrm{Yu}^{1, \mathrm{a}}$, Peng $\mathrm{Li}^{1, \mathrm{~b}}$ \\ ${ }^{1}$ Research Laboratory of Digital Factory, Shenyang Institute of Automation, Chinese Academy of \\ Sciences, Shenyang, 110016, China \\ aemail: yumiao@sia.cn, bemail: pengli@sia.cn
}

\begin{abstract}
Keywords: Aluminum Reduction Cell; Blackboard Structure; Expert System; Rule-Based Reasoning; Case-Based Reasoning
\end{abstract}

\begin{abstract}
Aluminum reduction cell is a non-linear, multivariable coupling time-varying system, inside which a large number of physical and chemical reactions occur. Meanwhile, aluminum reduction cell is often influenced by disturbance outside, which can easily cause instability of aluminum reduction cell. For the analysis of aluminum reduction cell, a kind of expert system based on blackboard structure is proposed, which can analyze aluminum reduction cell by combination of rule-based reasoning and case-based reasoning based on the data of blackboard structure and information from knowledge base. For an aluminum plant, experiment result has shown that expert system based on blackboard structure can analyze the aluminum reduction cell effectively, and it has certain engineering practicability.
\end{abstract}

\section{Introduction}

Aluminum reduction cell is a non-linear, multivariable coupling time-varying system, and material balance and energy balance inside the cell are changing constantly, which can lead to cold trough or hot trough. So it is particularly important to establish an expert system for the analysis of aluminum reduction cell [1].

For such a complex and dynamic process as aluminum electrolysis, it is difficult to establish a mathematical model to realize optimal control of production process. Expert system [2] shows the superiority in solving this kind of problem, which has achieved a wide range of applications as a very active branch of artificial intelligence [3]. Expert system does not rely on the mathematical model of system, and it solve the complex issues that need human expert to deal with by reasoning based on empirical knowledge [4] from experts in the field. So it can improve the accuracy and reliability of the analysis of aluminum reduction cell by use of expert system [5].

Expert system based on rule-based reasoning [6] and expert system based on case-based reasoning [7] are the two most common kinds. Expert system based on rule-based reasoning has strong explanatory as well as simple forms of expression [8], but the rules are difficult to be obtained. Expert system based on case-based reasoning can obtain answers to questions by case retrieval without rule match, but case coverage and system efficiency is a pair of irreconcilable contradictions [9]. To solve these problems, expert system based on blackboard structure is proposed, which can utilize the mechanism of combination of rule-based reasoning and case-based reasoning [10] to realize mutual compensation of advantages. Meanwhile, data from the solving process can be organized hierarchically by using blackboard structure [11], which can make sure the whole expert system works efficiently.

\section{Overall Structure of Expert System Based on Blackboard Structure}

Blackboard structure is an effective method for solving complex problems, and expert system based on blackboard structure is shown in Figure1, which is mainly composed of blackboard structure, inference engine, knowledge base, etc. Blackboard structure is composed of field blackboard and environment blackboard, which is also the platform for information transmission, knowledge collaboration and Parallel interaction. Inference engine makes inference by rule-based 
reasoning and case-based reasoning based on information from field blackboard and environment blackboard. Knowledge base provides cases and rules for the analysis of aluminum reduction cell.

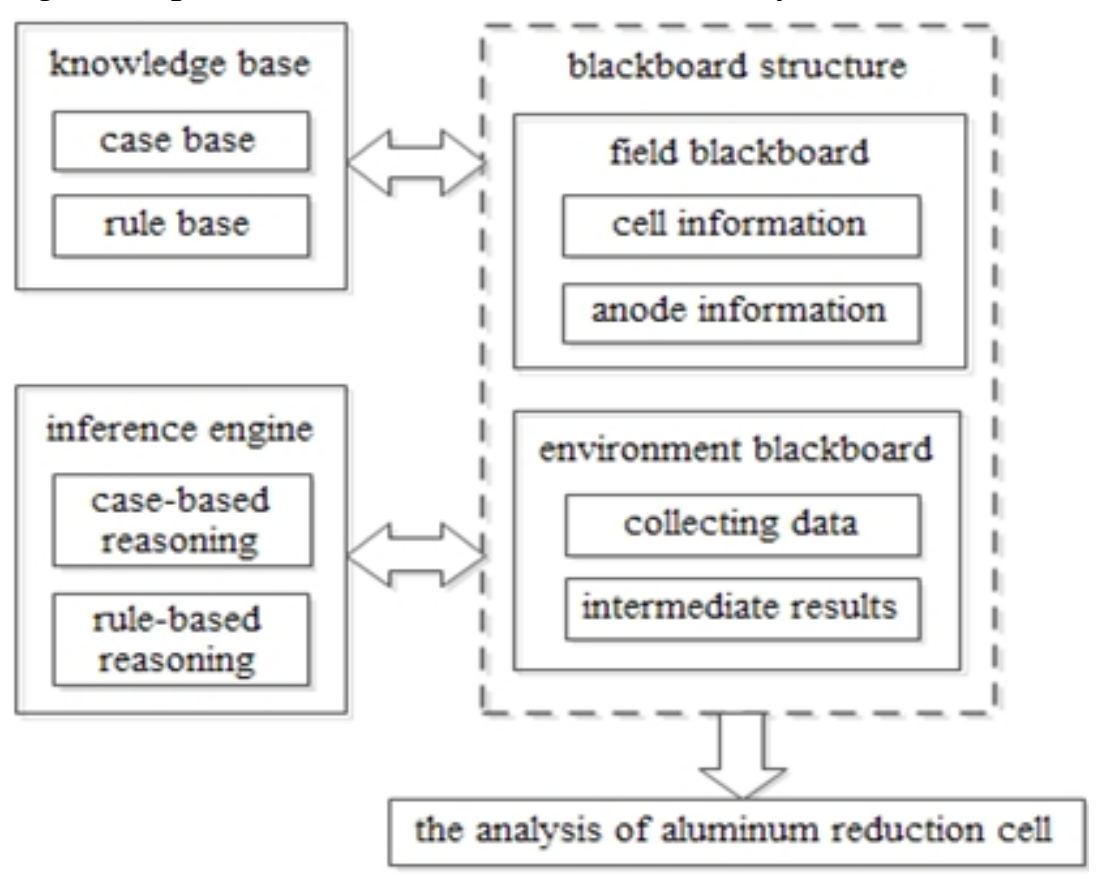

Fig. 1. Overall structure of expert system based on blackboard structure

\section{Blackboard Structure}

Blackboard structure is composed of field blackboard and environment blackboard. Field blackboard is used for storing basic information of aluminum reduction cell, which includes cell information and anode information. Environment blackboard is used for storing dynamic information during the operation of expert system, which includes collecting data from aluminum electrolysis process and intermediate results from inference engine.

\section{Knowledge Base}

Knowledge base is used for storing cases and rules for the analysis of aluminum reduction cell, which is composed of case base and rule base.

The core of building case base is to store historical cases into case base according to the representation of case, which come from the retrieval of daily record of aluminum electrolysis process. Case base is composed of cases, and the case includes the name of case, the date of case, the result of case, the solving measures of case and the characteristic parameters of case.

Rule base uses the representation of production rule based on syntax analysis, which can realize complex mathematical operations better.

In the representation of production rule based on syntax analysis, the condition of rule supports variable expression with comparison, which is described as follows: <varExpression operator [varExpression | constant]>. "VarExpression" represents variable expression, whose values come from environment blackboard. "Operator" represents comparison including “>”, ">=”, “<”, “<=” and "=”. "[varExpression | constant]" represents varExpression or constant. Workflow of the representation of production rule based on syntax analysis is shown in Figure2. Firstly, varExpression is written based on the arithmetic relations of variables by user. Furthermore, variables are extracted from varExpression based on regex, and then the extracted variables are replaced with the corresponding values from environment blackboard. Finally, numerical solution of varExpression is carried out. 


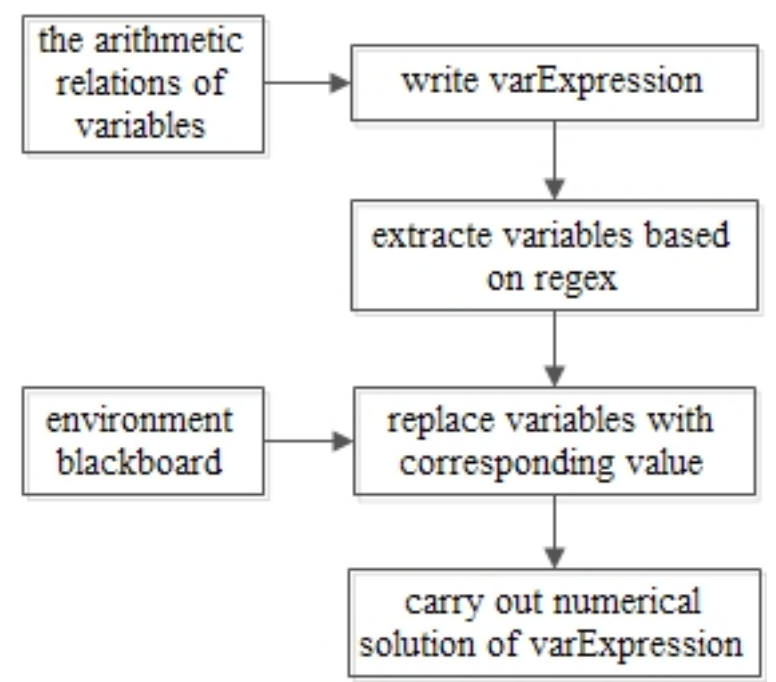

Fig. 2. Workflow of the representation of production rule based on syntax analysis

\section{Inference Engine}

Inference engine makes inference by combination of rule-based reasoning and case-based reasoning, and its workflow is shown in Figure3. Firstly, case-based reasoning that mainly matches the target event with cases of case base is carried out based on environment blackboard data. If case match is successful, solving results are given and the reasoning is finished. Otherwise, rule-based reasoning is carried out by way of forward reasoning. Then, if forward reasoning is successful, solving results are given and the reasoning is finished. Otherwise, the reasoning is finished.

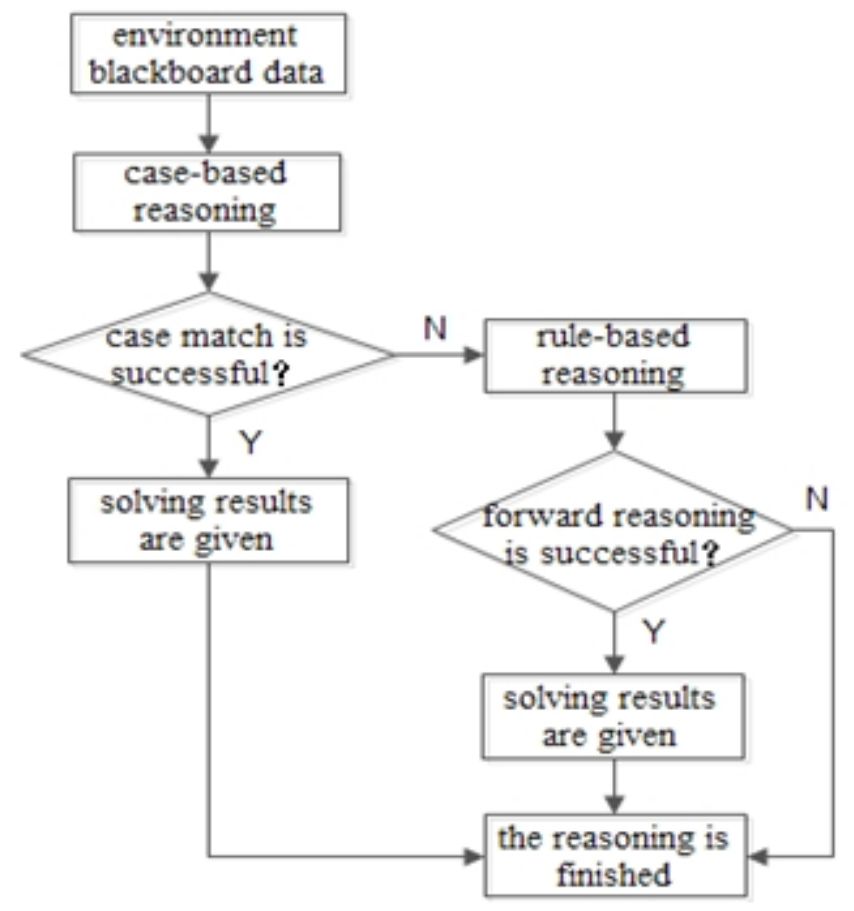

Fig. 3. Workflow of the representation of inference engine

\section{Test results}

An aluminum plant with 300kA electrolyzer is selected as the application case based on the expert system this paper proposed. The analysis of aluminum reduction cell in the aluminum plant is because it is lack of experienced workers and the cell analysis contains many subjective factors. For the above problems, the workflow of expert system this paper proposed is as follows.

Firstly, the case base is established, and there are only two cases listed as follows due to the 
limited space of the paper, which are "Case1" and "Case2":

Case1\{ name: hot trough; date: 2013-2-12; result: occurrence of hot trough; solving measures: add cryolite to cell; haracteristic parameters: \{molecular ratio: 2.9, aluminum level: 20$\}$;\}.

Case2 2 name: cold trough; date: 2013-3-12; result: occurrence of cold trough; solving measures: raise operating voltage haracteristic parameters: \{molecular ratio: 2.1, aluminum level: 26$\}$; .

Then the rule base is established. Al represents aluminum level. $C R$ represents molecular ratio. There are two rules as follows according to the aluminum plant due to limited space of the paper, which are "Rule1" and "Rule2":

Rule1: IF CR $>2.78$ and $A l<21$ THEN hot trough

Rule2: IF CR $<2.11$ and $\mathrm{Al}>26$ THEN cold trough

Finally, the cell analysis of expert system is carried out with the monitoring data of the aluminum plant as input, which include molecular ratio data and aluminum level data. The monitoring data of the aluminum plant for input are shown in Figure4.

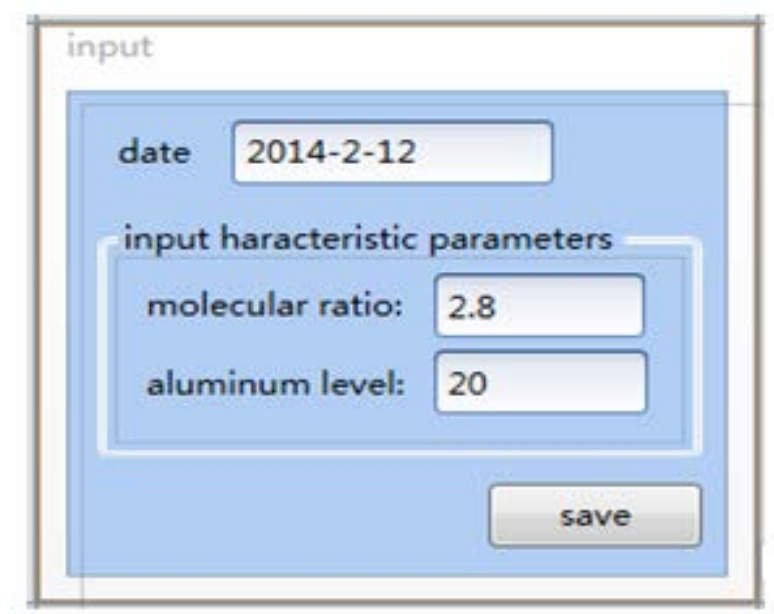

Fig. 4. Input interface of the monitoring data of the aluminum plant

Then we get the result "hot trough" after the inference by combination of rule-based reasoning and case-based reasoning based on knowledge base. "Case1" above and "Rule1" above both recommend the result "hot trough" for the industrial site, and the interface of the results is shown in Figure5. The validity of the result has been approved by technicians, and measures have been taken to solve the problem, which proves that the expert system this paper proposed can make effective analysis of aluminum reduction cell.

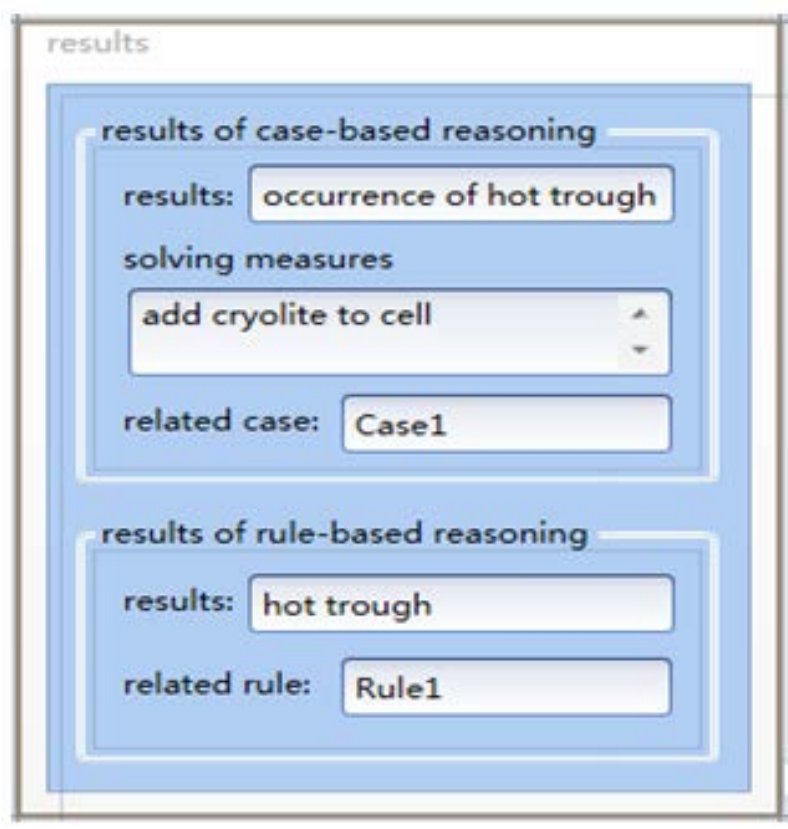

Fig. 5. Interface of the results 


\section{Conclusion}

Expert system for the analysis of aluminum reduction cell is developed based on blackboard structure. Through the cell analysis of an aluminum plant with 300kA electrolyzer, it has proved that the expert system can make effective analysis of aluminum reduction cell so as to solve the problem of the lack of experienced workers.

On the basis of the study above, we still need to do further study as follows: (1) Further research need to be done with the mechanism of aluminum electrolytic process, which is valuable for the enrichment of knowledge base. (2) expert system has strong explanatory, but it also has poor applicability for the system with little priori knowledge. By combining with neural networks, the expert system can do much better for unknown faults.

\section{Acknowledgement}

This work was financially supported by National High-tech R\&D Program of China (863 Program) (2013AA040705).

\section{References}

[1] Kristian Etienne Einarsrud, Stein Tore Johansen. Modelling of bubble behaviour in aluminium reduction cells[J]. Progress in computational fluid dynamics, 2012, 12(2/3):119-130

[2] E.F.Carrasco, J.Rodrguez and A.Punal: Expert Systems with Applications Vol. 22(2002), p.11-20

[3] Jianwen Guo, Dejie Yu and Jian Liu: Computer Engineering Vol. 36(2010), p. 22-24 In Chinese

[4] Shi-Kuo Chang,Wu-Haung Cheng,A Methodology for Structured Database Decomposition[J]. Software Engineering, IEEE Transactions on,1980

[5] S. P. Lu,L. Tikasz,R. T. Bui et al.Aluminum electrolysis process diagnosis by expert system[C]. =//Light Metals 2000.2001:55-68.

[6] Kim, Miryung,Notkin, David,Grossman, Dan et al.Identifying and Summarizing Systematic Code Changes via Rule Inference[J].IEEE Transactions on Software Engineering,2013,39(1):45-62.

[7] Ernesto Ocampo,Mariana Maceiras,Silvia Herrera et al.Comparing Bayesian inference and case-based reasoning as support techniques in the diagnosis of Acute Bacterial Meningitis[J].Expert Systems with Application,2011,38(8):10343-10354.

[8] Ramesh T S, Davis J F, Schwenzer G M. Knowledge-based diagnostic systems for continuous process operations based upon the task framework. Computers and Chemical Engineering, 1992,16(2):109-127

[9] Fu, Wai-Tat,Dong, Wei.Collaborative Indexing and Knowledge Exploration: A Social Learning Model[J].IEEE intelligent systems,2012,27(1):39-46.

[10]Carlos Alberto Costa,Marcos Alexandre Luciano,Celson Pantoja Lima et al.Assessment of a Product Range Model concept to support design reuse using rule based systems and case based reasoning[J].Advanced engineering informatics, 2012, 26(2):292-305.

[11]Y.C. Jiang,Z.Y. Xia,Y.P. Zhong et al.An adaptive adjusting mechanism for agent distributed blackboard architecture[J].Microprocessors and Microsystems, 2005, 29(1):9-20. DOI:10.1016/j.micpro.2004.06.001 